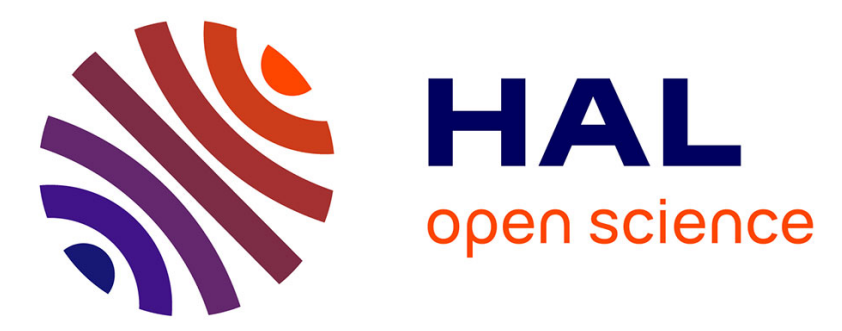

\title{
Qualitative and quantitative assessment of water sorption in natural fibres using ATR-FTIR spectroscopy
}

Amandine Célino, Olivier Gonçalves, Frédéric Jacquemin, Sylvain Fréour

\section{To cite this version:}

Amandine Célino, Olivier Gonçalves, Frédéric Jacquemin, Sylvain Fréour. Qualitative and quantitative assessment of water sorption in natural fibres using ATR-FTIR spectroscopy. Carbohydrate Polymers, 2014, 101, pp.163-170. 10.1016/j.carbpol.2013.09.023 . hal-01007227

\section{HAL Id: hal-01007227 \\ https://hal.science/hal-01007227}

Submitted on 13 Mar 2018

HAL is a multi-disciplinary open access archive for the deposit and dissemination of scientific research documents, whether they are published or not. The documents may come from teaching and research institutions in France or abroad, or from public or private research centers.
L'archive ouverte pluridisciplinaire HAL, est destinée au dépôt et à la diffusion de documents scientifiques de niveau recherche, publiés ou non, émanant des établissements d'enseignement et de recherche français ou étrangers, des laboratoires publics ou privés. 


\title{
Qualitative and quantitative assessment of water sorption in natural fibres using ATR-FTIR spectroscopy
}

\author{
A. Célino ${ }^{1 *}$, O. Gonçalves ${ }^{2}$ F. Jacquemin ${ }^{1}$, S. Fréour ${ }^{1}$ \\ ${ }^{1}$ Institut de Recherche en Génie Civil et Mécanique, UMR CNRS 6183, 37 Boulevard de \\ l’Université, 44602 Saint-Nazaire Cedex, France, \\ ${ }^{2}$ GEPEA, UMR CNRS 6144, 37 Boulevard de l'Université, 44602 Saint-Nazaire Cedex, \\ France \\ *Corresponding author: Amandine Célino \\ GeM, UMR CNRS 6183 \\ 37 Boulevard de l’Université, BP 406 \\ 44602 Saint-Nazaire Cedex
}

Telephone number: +33240172625

Fax number: +33240172618

E-mail address: amandine.celino@univ-nantes.fr

\begin{abstract}
In the field of composite materials, natural fibres appear to be a viable replacement for glass fibres. However, in humid conditions, strong hydrophilic behavior of such materials can lead to their structural modification. Then, understanding moisture sorption mechanisms in these materials is an important issue for their efficient use. In this work, the water sorption on three natural fibres (flax, hemp and sisal) was studied using Fourier Transformed InfraRed spectroscopy. The spectral informations allowed both qualitative and quantitative analysis of the moisture absorption mechanisms. The main chemical functions involved in the water sorption phenomenon were identified. The absolute water content of the fibres was also determined by using a Partial Least Square Regression (PLS-R) approach. Moreover, typical sorption isotherm curves described by Park model were fitted as well as water diffusion kinetics These last applications confirmed the validity of the FTIR spectra based predictive models.
\end{abstract}

Keywords: natural fibres, water sorption, Fourier Transformed InfraRed spectroscopy, Partial Least Square Regression, diffusion kinetics

\section{Introduction}

Nowadays, natural fibres are considered to be a good alternative for glass fibres replacement in the purpose of composite material reinforcement (particularly in automotive manufacturing or sport and leisure sector) (Bledzki \& Gassan, 1999; Suddell \& Evans, 2005). These fibres 
present a better environmental impact than glass fibres (recyclability, biodegradability) as well as higher specific mechanical properties because of their low density. However, their pronounced hydrophilic behavior -due to their particular structure- leads to high level of moisture absorption in wet environments (Célino, Fréour, Jacquemin, \& Casari, 2013). This results in the structural modification of the fibres and an evolution of their mechanical properties together with the composites in which they are fitted in (Dakhal, Zhan, \& Richardson, 2007; Placet, Cisse, \& Boubakar, 2012; Symington, Banks, David, \& Pethrick, 2009). Thereby, the understanding of these moisture absorption mechanisms is of great interest to get a better control of such new biomaterials.

Generally, one of the most important factors controlling the water diffusion phenomenon in polymeric materials is the molecular interaction occurring between the diffusing compound and the substrate. The diffusion phenomenon is subjected to the ability of the polymer molecular sites to establish hydrogen bonds with the water molecules. Spectroscopic techniques such as Nuclear Magnetic Resonance (NMR), dielectric or Fourier Transform Infra-Red spectroscopy (FTIR) have been proved to be well adapted to study this phenomenon since they allow to characterize molecular interactions involving potential sorption sites for water (Mijovic \& Zhang, 2003; Popineau, Rondeau-Mouro, Suplice-Gaillet \& Martin, 2005). Among these approaches, FTIR spectroscopy has been widely used to study water transport in polymer and particularly to study the water sorbed into epoxy resins (Cotugno, Larobina, Mensitieri, Musto \& Ragosta, 2001; Feng, Berger, \& Douglas, 2004; Fieldson \& Barbari, 1993; Musto, Ragosta, \& Mascia, 2000). Indeed, this technique provides attractive features: i.e. the very high sampling rate, the sensitivity, the accuracy of the quantitative analysis and the informations at the molecular level contained in the vibrational spectra. Moreover, the development of Attenuated Total Reflectance FTIR spectroscopy (ATR-FTIR) encouraged and facilitated the use of this non-invasive technique directly onto solid materials (Chalmers \& Dent, 1997).

On pure cellulosic polymers, the potential sorption sites for water were determined to be hydroxyl and carboxyl groups which are particularly easily detected in FTIR spectroscopy (Berthold, Olsson, \& Salmén, 1998). However, few studies have been performed to characterize water sorption by FTIR directly on raw lignocellulosic fibres. (Laity \& Hay, 2000) demonstrated that it was possible to reproduce water sorption kinetics by recording infrared spectra in reflexion mode on cellophane. More recently, (Olsson \& Salmén, 2004) examined the association of water on pulp paper using FTIR spectra acquired in transmission mode. Their results indicated the existence of characteristic bands affected by water. Moreover they determined a linear relationship between the absorbance of those bands and the water content, using a univariate approach. This last work was of particular importance since it highlighted major features helping the understanding of chemical groups involved in water diffusion in pulp paper. However both approaches, based on univariate analysis of FTIR signal failed to properly reproduce the sorption isotherms.

The aim of this work is the use of FTIR as an experimental tool to investigate the water sorption onto raw plant fibres, known to be good candidates for the reinforcement of 
composite materials (i.e. flax, hemp and sisal). First FTIR spectral signature of each fibre was investigated in order to describe the molecular effect of the water sorption mechanisms. Secondly, a multivariate model linking water sorption and the whole FTIR spectra was developed using partial least square regression (PLS-R). Finally this model was applied for the accurate monitoring of the water diffusion in the tested biomaterials.

\section{Materials and methods}

\subsection{Materials}

Among the disposable plant fibres, hemp, flax, and sisal fibres were chosen because they presented the best mechanical properties regarding the replacement of glass fibres for the purpose of reinforcing the polymeric matrix (Summerscales, Dyssanayake, Virk \& Hall, 2010; Wambua, Ivens, \& Verpoest, 2003,). In our study, the bundle of the model fibres was investigated. Bundle of fibres are extracted from the stem (flax and hemp) or the leaf (sisal) of the plant. They are composed of about ten elementary fibres linked together by a pectic cement (i.e. the middle lamella). Their section is in the order of a few millimeters.

\subsection{Moisture sorption protocol}

First, all thefibre samples tested were dried in desiccators containing silicate gel for 48 hours before controlled moisture sorption treatment. Then, samples were placed in different hygroscopic conditions in a climatic chamber (supplied by Climats and assisted by Spiral 3 software) in order to get samples with different water content. The relative humidities tested were 30, 50, 60, 75, 85 and $97 \%$ at room temperature. Experimental sorption isotherms and sorption kinetics were obtained by periodically weighting the samples. Dynamic Vapour Sorption apparatus could also been used to plot sorption isotherms with more accuracy as demonstrated by (Bessadok et al., 2009 or Xie et al., 2011). But in our case, gravimetric measurement provided necessary results. Data were read to $0.01 \mathrm{mg}$ on a precision balance (Sartorius - MC1 Analytic AC210P). The equilibrium moisture content Ms is considered to be reached when the mass is stable according to relation (1).

$$
\mathrm{M}_{\mathrm{W}}(\%)=\frac{\mathrm{M}(\mathrm{t})-\mathrm{M}_{0}}{\mathrm{M}_{0}} \times 100
$$

where $\mathrm{M}_{0}$ is the initial weight of the bulk specimens before moisture sorption (dry conditions) and $\mathrm{M}(\mathrm{t})$ is the weight of the specimen at time $\mathrm{t}$.

\subsection{Infrared spectroscopic investigations}

\subsubsection{ATR-FTIR spectra acquisition}

Infrared spectra were recorded in reflection mode directly on the single reflexion diamond crystal of the ATR accessory loaded with bundles of the three fibres presented in section 2.1. The Bruker tensor 27 FTIR spectrometer equipped with the ATR platinum module, with a deuterated triglycine sulphate detector RT-DLaTGS and the OPUSv7.0.122 software (Bruker Optics, Germany) was set up with the following parameters. The spectral resolution was fixed 
126 to $1 \mathrm{~cm}^{-1}$, the number of scans to 32, the selected spectral range between 4000 and $400 \mathrm{~cm}^{-1}$. 127 The penetration depth in the fibres was $0.4 \mu \mathrm{m}$ at $4000 \mathrm{~cm}^{-1}$ and $2.7 \mu \mathrm{m}$ at $600 \mathrm{~cm}^{-1}$ according 128 to equation (2) relating penetration depth (dp) to wavelength.

$$
\mathrm{dp}=\frac{\lambda}{2 \Pi \mathrm{n}_{1} \sqrt{\sin ^{2} \theta-\left(\mathrm{n}_{2} / \mathrm{n}_{1}\right)^{2}}}
$$

129 Where $\lambda$ is the infrared wavelength, $\mathrm{n}_{1}$, the refractive index of the internal reflexion element 130 (IRE), $\mathrm{n}_{2}$ the refractive index of the substrate and $\theta$ the angle of incidence of the IR beam 131 (Griffith \& Haseth, 2007). In our case $\mathrm{n}_{1}=2.41, \mathrm{n}_{2}=1.5$ for the fibres assimilated as polymer, $132 \theta=40^{\circ}$, and $\lambda=4000-600 \mathrm{~cm}^{-1}$ ).

133 Background spectra were collected using the same instrument settings as those employed for 134 the samples and was performed against air. Spectra were recorded for 10 replicates per fibre 135 sample.

136

137

138

139

140

141

142

143

144

145

146

147

148

149

150

151

152

153

154

155

156

157

158

159

160

161

162

163

164

\subsubsection{ATR-FTIR spectra acquisition in kinetic mode}

For the monitoring of water diffusion, time dependent ATR-FTIR spectra were acquired every 2 minutes in ambient conditions during drying of a saturated fibre sample. Each tested fibres were systematically placed in a relative humidity atmosphere of $95 \%$ before the measurement. Recorded spectra were directly treated by Partial Least Square regression (section 2.3.4) in order to determine the water content and to plot the drying kinetics of the tested fibres. Then, the results were compared with the kinetics obtained by the gravimetric measurements. For the gravimetric measurements, the samples were placed on a precision balance $(0.01 \mathrm{mg})$ during the drying step and data were collected every 2 minutes.

\subsubsection{ATR-FTIR spectra preprocessing}

All the spectra were recorded to the background spectra. For the PLS-R model calibration and validation the raw spectra were used since OPUS v 7.0.122 software was set up to manage spectral corrections automatically, according to the value of its optimization criteria. For the Kruskal Wallis analysis the raw spectra were corrected for ambient $\mathrm{CO}_{2}$ only and smoothed. For the multivariate analysis, the raw spectra were corrected for ambient $\mathrm{CO}_{2}$, smoothed and a second derivative calculation was also performed. All these treatments were achieved using integrated functions of (Bruker Optics, Germany)

2.3.4 Qualitative Multivariate analysis of ATR-FTIR spectra

Pre-processed spectra or pre-processed second derivative spectra were exported as text files for file format modification on Microsoft Excel v14.0.0 or statistical treatment on R 2.15.2. Second derivative spectra were systematically used to improve the infrared band resolution and thus enhance the discrimination of vibrators contributing to the shape of raw FTIR spectra (Mecozzi, Pietroletti, \& Tornambe, 2011). The spectral data were centered and scaled (mean subtracted and divided by standard deviation) to minimize the unit range influence before performing unsupervised multivariate analysis (Principal Component Analysis (PCA) and Hierarchical Clustering on Principal Components (HC-PC)) using the factominer R package (Lê, Josse \& Husson, 2008). The number of classes was determined without a priori by firstly 
using the unsupervised multivariate analysis (i.e. PCA). A refined assessment of the clusters was then performed using a Partial Least Square Discriminant Analysis (PLS-DA), conducted on all the wave numbers stemming from the ATR-FTIR spectra. This multivariate approach was used in order to enhance the formation of clusters by using a model of class prediction (for review see Wold, Sjöström, \& Eriksson, 2001). The quality of the prediction was characterized by two parameters, $\mathrm{R}^{2}(\mathrm{Y})$ (goodness of the fit) and $\mathrm{Q}^{2}$ (predictive capability of the model). The clustering capabilities of the PLS-DA model were considered as satisfactory with both $\mathrm{R}^{2}(\mathrm{Y})$ and $\mathrm{Q}^{2}$ values superior to 0.5 (Westerhuis et al., 2008). The generation of the 173 PLS-DA models was performed in two steps: first the calibration step $\left(\mathrm{R}^{2}(\mathrm{Y})\right.$ and $\left.\mathrm{Q}^{2}\right)$, second 174 the validation step named also the cross validation step $\left(\mathrm{R}^{2}(\mathrm{Y})\right.$ int and $\mathrm{Q}^{2}$ int). For the cross validation step, (Westerhuis et al., 2008) latest study indicated clearly that it was important to notice that even with negative $\mathrm{Q}^{2}$ int values, it was still possible to obtain clear separation between classes. The PLS-DA analysis was conducted using the web server analysis pipeline Metaboanalyst 2.0, dedicated to metabolomic data exploitation (Xia, Mandal, Sinenikov, Broadhurst, \& Wishart, 2012). For this supervised multivariate analysis ATR-FTIR spectral data were $\log 10[1+\mathrm{x}]$ - transformed and Pareto scaled (divided by the square root of the standard deviation) (Van den Berg, Hoefsloot, Westerhuis, Smilde, \& Van Der Werf, 2006). To assess the influence of the water content on the ATR-FTIR spectra of the tested fibres, a Kruskal Wallis Test (non parametric ANOVA) was performed per wave number of the ATRFTIR raw spectra recorded on a tested fibre for different water contents. As this non parametric version of the ANOVA does not make any assumption on the nature of the underlying distribution (it does not assume the distribution to be normal), it should be less sensitive to the outliers commonly found in FTIR signatures (since the outliers are taken into account). This allowed a better detection of the vibrators impacted by the water uptake (Di Giambattista et al., 2011). P-value beyond significance threshold (0.05) were plotted on raw

\subsubsection{Quantitative analysis of ATR-FTIR spectra}

194 There are two steps to obtain PLS-R models: the calibration and the validation. Generally two 195 kinds of validation can be performed: the cross validation and the external validation. Usually 196 the calibration and the cross validation are performed in a single step since identical data set is used to perform calculation.

198

\section{(i) Calibration and cross validation}

200 For the calibration (cross validation) step, the procedure of calculation and selection of the 201 best predictive model was iterative and comprised a loop formed of two steps. Firstly the pre202 processed infrared spectra were regressed against the reference value (i.e. for a considered 203 fibre: its water content determined gravimetrically). Secondly a full cross validation of the 204 model was performed by omitting one sample and operating the test in the remaining spectral 205 data set. This procedure allowed finding models with high correlations $\mathrm{R}^{2}$ and low Root Mean 206 Square Error of Cross-Validation (RMSECV). The infrared data sets used in our study 
comprised 11 samples and 5 replicates per sample, i.e. 55 spectra (ASTM Committee E13, 208 2005).

209 (ii) External validation

210 An independent data set was used for the model evaluation (4 samples and 5 replicates per 211 sample, i.e. 20 spectra) in order to obtain highest correlations (i.e. high $\mathrm{R}^{2}$ ) and lowest root 212 mean square error of prediction (RMSEP).

\section{Results and discussion}

2153.1 FTIR qualitative analysis of the water uptake effect on the studied fibres

\section{3.1.1 Each model fibre presented its own FTIR fingerprint}

217 The second derivative spectral data were qualitatively analysed using Partial Least Square 218 Discriminant Analysis (PLS-DA) for each model fibres and for three different relative 219 humidities ( $\mathrm{RH}=10,50$ and $97 \%$ ). On figure 1, results indicate that the three fibres are 220 clustered into three distinct groups, clearly associated to each type of fibre (axis 1). Inside 221 each cluster, it is moreover possible to distinguish subgroups according to relative humidity 222 content (axis 2).

223 The clustering results clearly reveal that each type of fibre could be distinguished according to 224 its FTIR spectrum, exhibiting moreover differences for the three tested relative humidities. 225 Those observations suggest that each fibre presents its own FTIR signature, depending on its 226 proper chemical composition. Indeed, according to the work of Satyanarayana and colleagues 227 (Satyanarayana, Arizaga, \& Wypych, 2009) this spectral signature could be directly related to the amount of the constitutive macromolecules (cellulose, hemicelluloses, lignin and pectin) 229 and to the crystallization degree of the cellulose which are both fibre dependent. Moreover, 230 as the chemical make-up and the crystallinity degree of the fibres are known to influence the 231 moisture sorption, the PLS-DA results suggest that each fibre should present its own water 232 diffusion behavior. Taken together all those remarks indicate that the water quantification 233 models should be developed per type of fibres. 


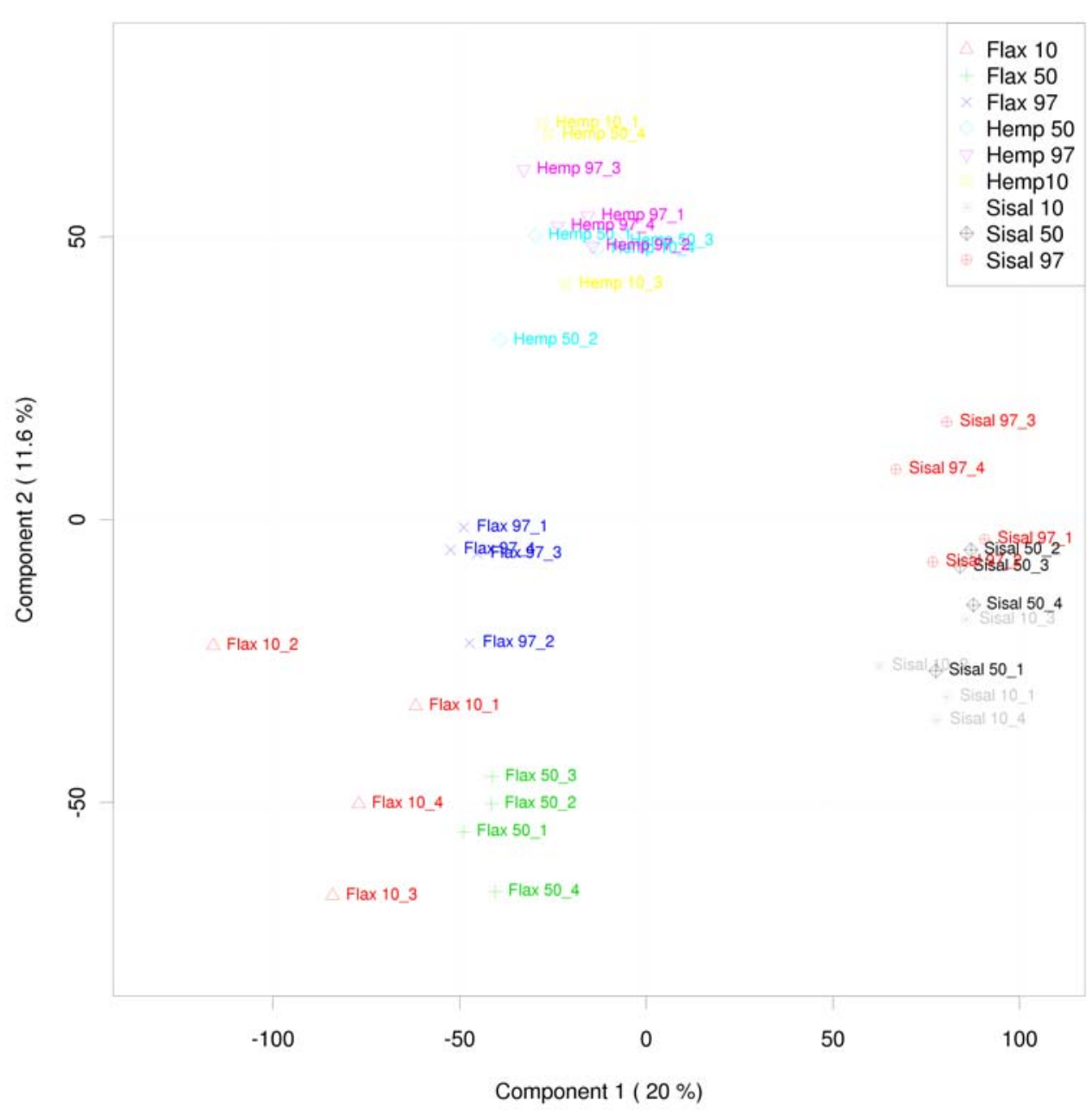

Figure 1: PLS-DA score plot of second derivative FTIR signatures of the three model fibres 237 for three $\mathrm{RH}$ conditions.

238 The legend in the upright corner details the nature of the model fibre and the values of the 239 tested $R H \%$ (as suffixes). The classification model used here presented the following 240 characteristics i.e. $R^{2}(Y)=0,989$ and $Q^{2}=0,869$ and was validated using 20 permutations $241 \quad\left(R^{2}(Y)\right.$ int $=0,555$ and $\left.Q^{2} i n t=-0,343\right)$.

242 3.1.2 Water sorption molecular sites could be identified for the model fibres subjected to 243 increasing $\mathrm{RH} \%$

244 The spectral dependence on the water content is illustrated on figure 2. The development of 245 the sisal FTIR spectra by increasing relative humidity and water content has been treated here 246 solely for reason of clarity. Similar results were observed for hemp and flax fibres (data not 247 shown). The results of the Kruskal Wallis analysis performed on each individual wave 248 number of the raw spectra highlight several zones of the sisal FTIR fingerprint that were 249 strongly impacted by the increasing water uptake. It helps the FTIR spectra bands assignation 250 (using table 1) and allows the interpretation of the experimental observations.

Table 1: Assignment of the main absorption bands in FTIR spectra of sisal, flax and hemp fibres. Sisal FTIR data were interpreted according to investigations issued from (Adibi, 
254

255

256

257

258

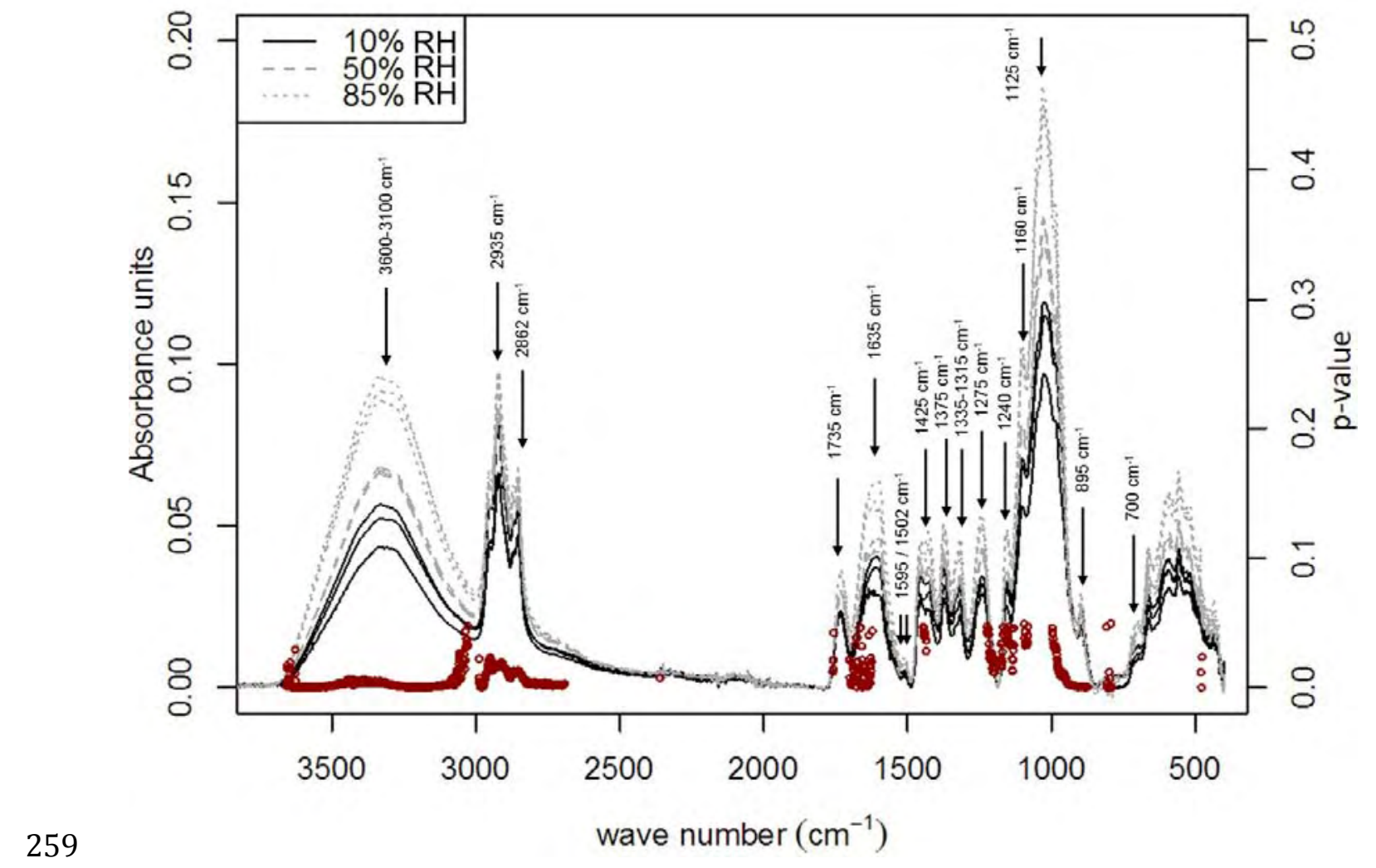

259

260

Cabrales, \& Haigler, 2013; De Rosa, Kenny, Puglia, Santulli, \& Sarasini, 2010; Liang \& Marchessault, 1959; Nelsson \& O’Connor, 1964).

\begin{tabular}{|c|l|}
\hline Wave number $\left.\mathbf{~ c m}^{-1}\right)$ & \multicolumn{1}{c|}{ Assignment } \\
\hline $\mathbf{3 6 0 0 - 3 1 0 0}$ & $\begin{array}{l}\text { Hydrogen bonded of OH stretching in cellulose } \\
\text { and/or hemicelluloses }\end{array}$ \\
\hline $\mathbf{2 9 3 5}$ & CH stretching of cellulose and hemicelluloses \\
\hline $\mathbf{2 8 6 2}$ & $\begin{array}{l}\text { CH } \text { stretching of cellulose and hemicelluloses } \\
\text { C=O stretching vibration of carboxylic acid in } \\
\text { pectin or ester group in hemicelluloses }\end{array}$ \\
\hline $\mathbf{1 7 3 5}$ & $\begin{array}{l}\text { OH bending vibration characteristic of sorbed } \\
\text { water }\end{array}$ \\
\hline $\mathbf{1 6 3 5}$ & Aromatic ring in lignin \\
\hline $\mathbf{1 5 9 5}$ & Aromatic ring in lignin \\
\hline $\mathbf{1 5 0 2}$ & Carboxylic acid of pectin and COO- vibration \\
\hline $\mathbf{1 4 2 5}$ & CH bending of cellulose and hemicelluloses \\
\hline $\mathbf{1 3 7 5}$ & CH ${ }_{2}$ wagging of cellulose and hemicelluloses \\
\hline $\mathbf{1 3 3 5 - 1 3 1 5}$ & Characteristic peak of lignin \\
\hline $\mathbf{1 2 7 5}$ & C-O of acetyl in pectin or hemicelluloses \\
\hline $\mathbf{1 2 4 0}$ & anti-symmetrical deformation of the C-O-C band \\
\hline $\mathbf{1 1 6 0}$ & C-O stretching and ring vibrational modes \\
\hline $\mathbf{1 1 2 5 - 8 9 5}$ & Characteristic of $\beta$-links in cellulose \\
\hline $\mathbf{8 9 5}$ & O-H out of plane bending \\
\hline $\mathbf{7 0 0 - 6 5 0}$ &
\end{tabular}

\section{8}


For clarity, only sisal fibre has been presented and only three relative humidities are shown

262 for three replicates (10\%; $50 \%$ and $85 \%)$. p-values scores, indicating significant impact of

263 the water uptake on the FTIR bands, were marked using red dots.

264

265

(i) Part of the water-fibre interactions could be associated to the formation of hydrogen bonds between the water and the hydroxyl groups of the cellulose or hemicelluloses constitutive of the raw material.

The broad and unresolved band situated between 3600 and $3000 \mathrm{~cm}^{-1}$ was found to be significantly impacted by the increasing relative humidity content. Potential molecular sites of the polymers constituting the studied fibre could be identified here to explain the interactions with the water molecules. Indeed, this band is generally associated to the $\mathrm{OH}$ stretching vibrations and hydrogen bonds of hydroxyl groups found in cellulosic materials. However, the absence of clear structured shape makes difficult the assignment of this absorption band (Fengel, 1992; Marchessault, 1962). Recently, (Kondo, 1997) and (Hinterstoisser \& Salmèn, 1999) helped its resolution by using fitting deconvolution model and DMA-FTIR coupled analysis, respectively. The hydrogen bonds of hydroxyl groups in the 3600-3000 $\mathrm{cm}^{-1}$ wave number range were thus associated to general intramolecular and intermolecular hydrogen bonding and to free hydroxyl in cellulose macromolecule. This peak is also representative of the contribution of the free or the bound water linked to the substrate (Cotugno, Mensitieri, Musto, \& Sanguigno, 2005; Murphy \& Pinho, 1995; Musto et al., 2000). Here it could be the amorphous phase of the cellulose or the hemicelluloses of the material.

282

(ii) The water uptake could be also characterized by the monitoring of the interaction between the polymers constituting the raw fibre and the free water.

The interaction of the cellulose or the hemicelluloses molecular sites of sisal, with the free water is also suggested by the significant impact monitored for the FTIR band situated at $1635 \mathrm{~cm}^{-1}$. Indeed such modification was already observed in the case of water diffusion and absorption on cellulosic material, and was found to be characteristic of the free water sorbed on studied material (Laity \&Hay, 2000; Murphy \& Pinho, 1995). The existence of this interaction should be normally reinforced by the presence of a peak situated at approximately $700 \mathrm{~cm}^{-1}$ and assigned to the out of plane bending of the hydroxyl groups characteristic of the free water molecule. However this last peak is not clearly highlighted by the Kruskal Wallis analysis to be dependent of the water content, bringing therefore uncertainty about the influence of free water contribution monitoring. This lack of characterization could be 295 partially explained by the fact that in spectra recorded in attenuated total reflectance, the 296 definition between $700 \mathrm{~cm}^{-1}$ and $400 \mathrm{~cm}^{-1}$ could not be as good as in transmission mode, 297 precluding therefore the exploitation of the spectral information situated in this range of wave 298 numbers (Parker \& Roy, 1966).

(iii) Other molecular sites impacted by the increasing water content could be suggested. 
confirmed the previous observation of Olsson and colleagues (Olsson \& Salmén, 2004). The

305

306

307

308

309

310

311

312

313

314

315

316

317

318

319

320

321

322

323

324

325

326

327

328

329

330

331

332

333

334

335

336

337

338

339

340

341

342

343

344

345

346

evolution of the peak situated at $1425 \mathrm{~cm}^{-1}$ could also be associated to the interaction of the water to the carboxylic acid moiety of the pectin polymer as firstly depicted by Marchessault in his exhaustive study on wood polysaccharides infrared spectra (Marchessault, 1962). Surprisingly, according to the Kruskal Wallis p values assignment, the $\mathrm{CH}$ stretching bands situated at 2935 and $2900 \mathrm{~cm}^{-1}$ were found to be significantly impacted by the water uptake. This is difficult to interpret, since no clear chemical explanation could be envisaged. Several hypotheses could however bring elements of understanding. It could be inherent to the material which is known to be subject to chemical intrinsic variability or be assigned to nonspecific experimental variation. Indeed, tests have been performed on different raw fibres for the identical humidities and it effectively highlighted differences in this specific range of wave numbers (data not shown). The way the spectra were processing could also bring elements of explanation. Indeed they were analyzed with almost the same preprocessing treatment than the one used for the PLS-R model generation. In particularly they were not baseline corrected. The shoulder of the $\mathrm{OH}$ stretching vibration band clearly shifted up the whole $\mathrm{CH}$ bands when the amount of water increases in the sample -whereas no significant increase of the area was monitored (data not shown). This should perturb the Kruskal Wallis test in this particular zone, since it was performed per wave numbers and not per groups of wave numbers -i.e. a band. The combined use of a smoothing function or of moving average performed onto the p values results could enhance the pertinence of the Kruskal Wallis test. These last observations should be carefully considered in the forthcoming multivariate analysis interpretations.

\subsubsection{Summary}

The results presented above showed that it was possible to discriminate the type of fibre thru its ATR-FTIR second derivative signature using a PLS analysis. Moreover, results revealed that the whole fibre infrared spectra were impacted by the moisture changes. These features strongly suggest that to monitor accurately the water diffusion in the tested biomaterials, a multivariate approach should be used. Indeed, it should improve the water content prediction compared to a univariate approach since the spectral information linked with an increasing water content is visible on the whole spectrum.

\subsection{FTIR quantitative analysis of the water content of the studied fibres}

\subsubsection{It was possible to predict the water uptake of every tested fibre by developing material} dependent PLS-R models

The absolute water content of the tested fibres was determined by using a Partial Least Square Regression (PLS-R) approach. For each kind of fibre, the MIR spectra were used in the range from $4000 \mathrm{~cm}^{-1}$ to $400 \mathrm{~cm}^{-1}$ with the corresponding water uptake measured gravimetrically, to calculate multiple PLS-R models and to perform their validation. The models calculated from the information contained in the spectral regions situated between $3600 \mathrm{~cm}^{-1}$ to $3200 \mathrm{~cm}^{-1}$ and around $1650 \mathrm{~cm}^{-1}$ were selected preferentially since they were associated to the main regions characteristics of the interaction between the water and the polymers constituting each studied fibres (3.1). Other regions were not excluded since they could be associated to the 
347 material intrinsic variability (e.g. around $2900 \mathrm{~cm}^{-1}$ ). The automatic pre-processing 348 treatments, the spectral regions and the quality indicators (i.e. the cross-validation and the test 349 set validation results) used to calculate the PLS-R models are summarized in the table 2. The 350 spectral regions selected without a priori are in very good agreement with the observations 351 performed in 3.1, especially for the sisal fibre which presents a wave number range 352 distribution correlated to the three main components known to interact with the water 353 molecules. Moreover, the estimation of the errors associated to the predictive models, 354 highlights their pertinence since the RMSECV and RMSEP are very low ( $\leq$ to 1 ). The 355 average number of PLS components is inferior to 7 and the average coefficient of 356 determination greater than $96 \%$, confirming the quality of the selected models The 357 significance of these parameters has been well described in FitzPatrick and colleagues latest 358 works (FitzPatrick, Champagne \& Cunningham, 2012). The figure 3 illustrates the cross359 validation and the test set validation results obtained for the sisal fibre. The dispersion 360 diagrams, displaying the relationships between predictive moisture content obtained by PLS361 R quantitative model and gravimetric measurements show a very good correlation $\left(\mathrm{R}^{2}>98\right)$. 


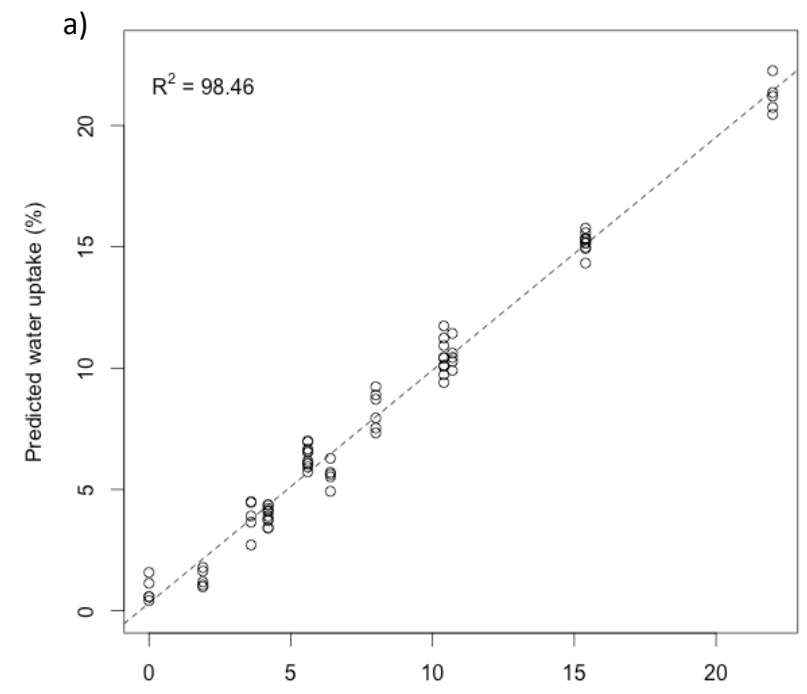

364

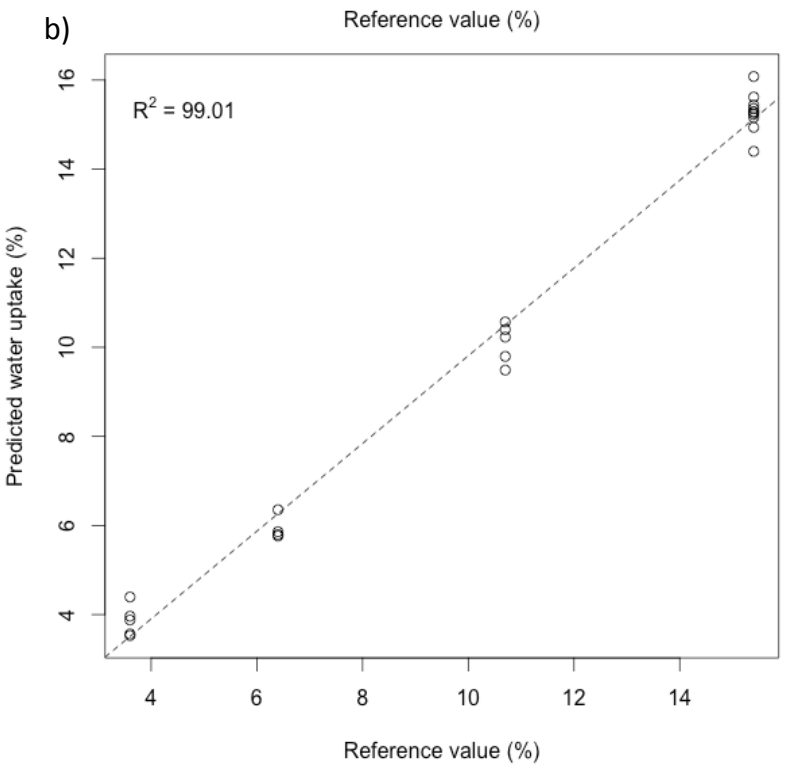

Figure 3: Cross-validation (a) and test set validation (b) results of water uptake prediction for sisal fibre.

The PLS-R predictions of the water content obtained with the FTIR approach were compared here to the gravimetric measurements. The water uptake is expressed in \% of the dry weight of the sisal fibre. The coefficients of determination (\%) of the regression lines (indicated in dotted lines) are indicated in the upper left corner. 
Table 2: PLS-R quality parameters for cross and test set validation for the models selected 384 for the three studied fibres.

385 RMSECV stands for Root Mean Square Error of Cross Validation. RMSEP stands for Root Mean Square Error of Prediction. $R^{2}$ is the coefficient of determination.

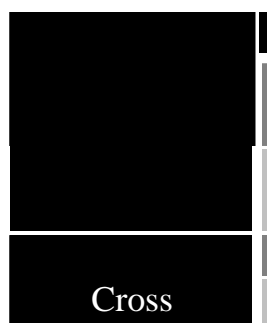

validation
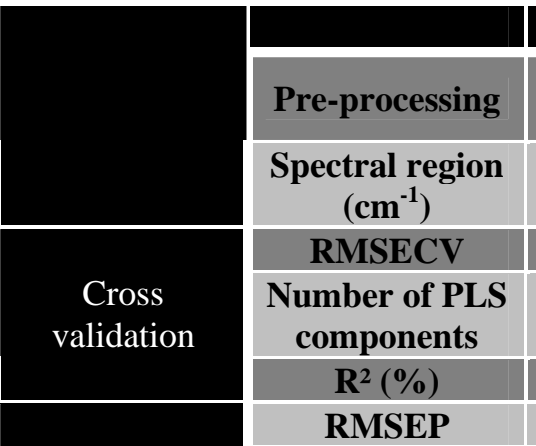

Hemp

Flax

Sisal

External

validation

\begin{tabular}{|c|c|}
\hline RMSECV \\
\hline $\begin{array}{c}\text { Number of PLS } \\
\text { components }\end{array}$ \\
\hline $\mathbf{R}^{2}(\%)$ \\
\hline RMSEP \\
\hline $\begin{array}{c}\text { Number of PLS } \\
\text { components }\end{array}$ \\
\hline $\mathbf{R}^{\mathbf{2}}(\%)$ \\
\hline
\end{tabular}

First derivative

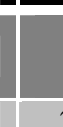

Min/max normalization

3998-1119/760-

3998-3277 / 2559-

2198 / 1839-400

1.14

(21

1.07

4

9

95.26

94.92

0.620

0.843

5

97.90

7

96.65

3.3 The calculated predictive models were applied to quantify the water sorption phenomenon

390 on the three tested fibres

\section{3.3.1 Monitoring of the sorption isotherm using PLS-R models}

392 The estimation of the water uptake of each studied fibre according to its relative humidity 393 exposition was performed using FTIR spectral information and gravimetric measurements as 394 reference method. FTIR data were interpreted in two ways, one using information arising 395 from one band characteristic of the water sorption (univariate approach) and one exploiting 396 the data of the whole FTIR spectra (multivariate approach: PLS-R model). First, the fibre 397 relative water content was estimated to be solely representative of the absorbance variation of 398 the hydroxyl groups situated in the 3600-3000 $\mathrm{cm}^{-1}$ range as already depicted by Olsson \& 399 Salmén (2004) (3.1.2). Second, the PLS-R models developed for each studied fibre were used 400 to predict the absolute water uptake taking into account the whole FTIR spectral information. 401 The sorption isotherms present a similar shape for the three studied fibres, whether they were 402 depicted by gravimetric measurements or deduced by FTIR data. The figure 4 illustrates the 403 case of the sisal and indicated that the water content is directly related to the relative 404 humidity by following a sigmoidal relation, as already described by (Alix et al., 2009; 405 Gouanvé, Marais, Bessadok, Langevin \& Métayer, 2007). That kind of sorption isotherms are 406 in a good agreement with the Park's model ( Park, 1986). This model assumes the association 407 of three mechanisms describing the three parts of the curve (figure 4). It is often used to 408 explain the sorption isotherms of hydrophilic and porous media, as cellulosic fibres (Bessadok 409 et al., 2009).

410 The first part of the curve could be related to Langmuir's mode ( $\mathrm{RH}<10 \%$, this mode is not 411 really visible on the figure 4). At these relative humidities, water is sorbed onto specific sites 412 by hydrogen bonding. As discussed in the section 3.1.2 (qualitative analysis of the bands 413 impacted by water) the specific sites could be hydroxyl functions of amorphous cellulose and 414 hemicelluloses or carboxylic function of pectin. When relative humidity increases, there is a 
saturation of these specific sites of sorption. Then, the water concentration increases linearly with relative humidity as Henry's law describes (until $\mathrm{RH}=65 \%$ ). This behavior could be explained by the porous structure of fibres where water is free to diffuse. The third part is well described by a power function that represents an aggregation phenomenon of water molecules. Indeed, at high relative humidity, water concentration is too important, and water molecules linked together to form clusters.

421 It is important to notice that the prediction arising from the FTIR PLS-R model fits very well the experimental gravimetric measurements $\left(R^{2}>99\right)$, highlighting the pertinence of the FTIR multivariate approach compared to the FTIR univariate method $\left(\mathrm{R}^{2}<90\right)$.

a)

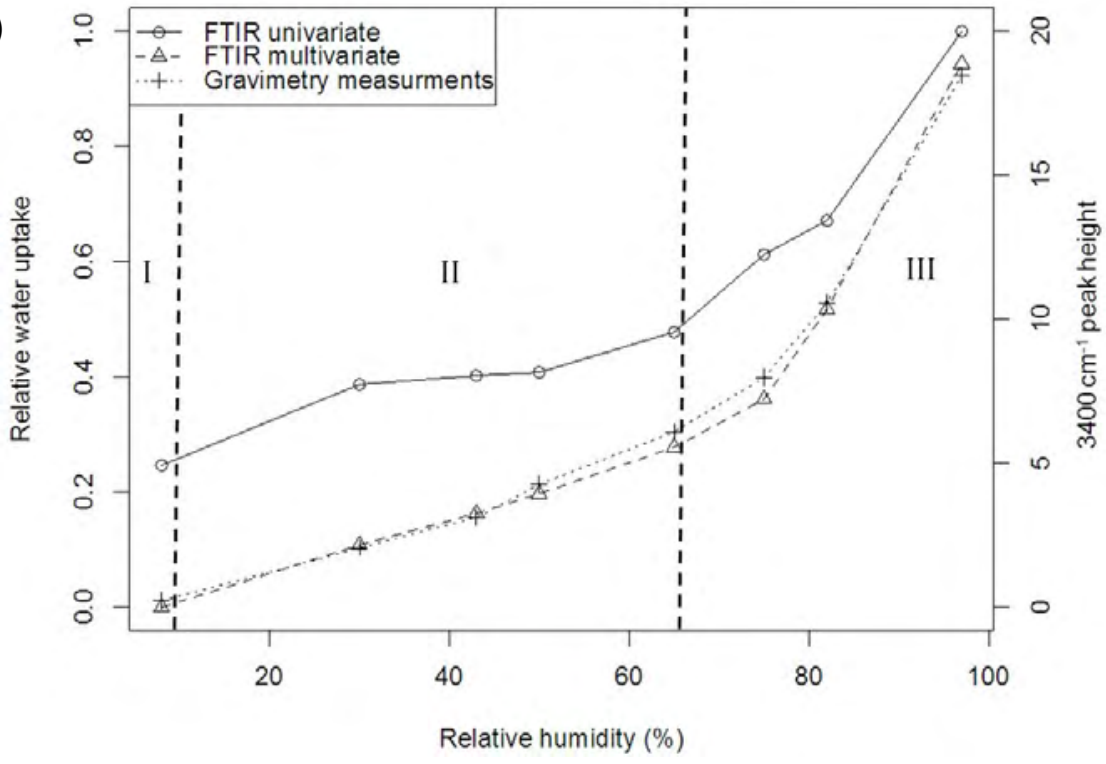

b)

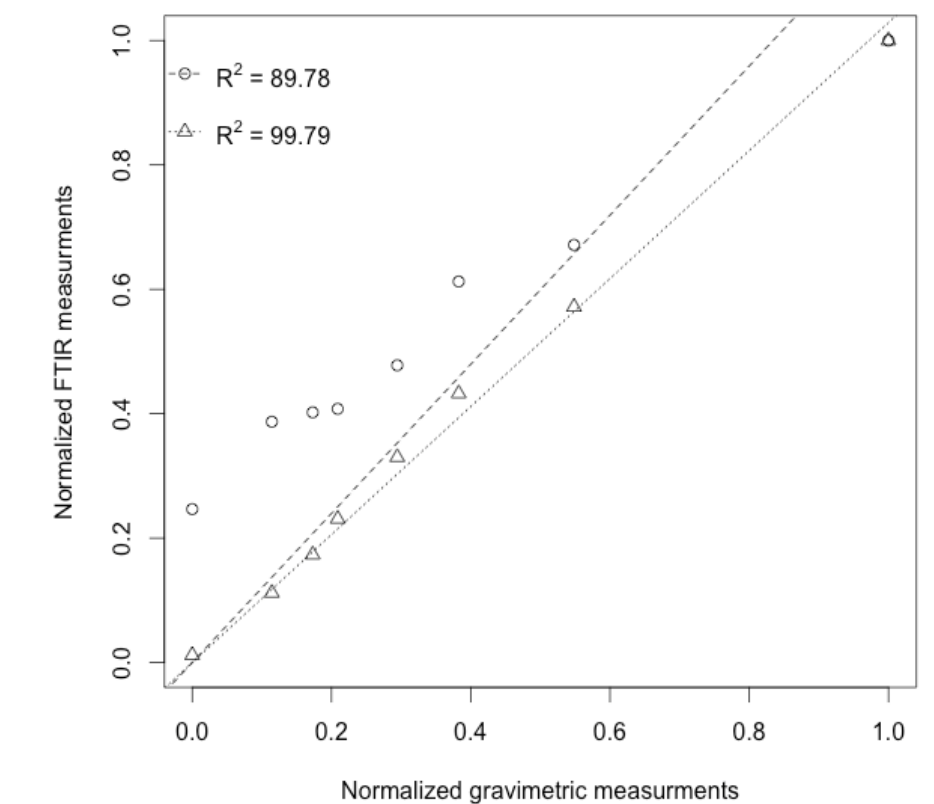

Figure 4: Comparison of sorption isotherm for sisal fibre using FTIR and gravimetric approaches. (a) Sorption isotherm plotted for the sisal fibre according to the measurement method (FTIR univariate, FTIR multivariate, gravimetry). (b) Comparison performed between 
normalized FTIR measurements and normalized gravimetric reference method measurements

432 (FTIR univariate-gravimetry: $O, F T I R$ multivariate-gravimetry $: \triangle$ ). $R^{2}$ stands for coefficient 433 of determination (\%).

434

435

436

437

438

439

440

441

442

443

444

445

446

447

448

449

450

451

452

453

454

455

456

457

458

459

460

461

462

463

464

465

466

467

468

469

470

471

472

473

474

\subsubsection{Monitoring of the diffusion kinetic using PLS-R models}

The desorption kinetics of the water sorbed by the studied fibres were also monitored by exploiting the multivariate FTIR approach. The fibre samples were first aged at RH $=95 \%$ until saturation was reached. Second, FTIR spectra were recorded in kinetic mode during their drying in ambient relative humidity conditions i.e. $\mathrm{RH}=45 \pm 3 \%$. FTIR spectral information was treated using the developed PLS-R models for each kind of studied fibres and compared to the gravimetric measurements, systematically performed in parallel. For reason of clarity, the desorption kinetics of sisal fibre is solely represented on figure 5a. FTIR data, predict that after moisture sorption in a wet environment at $\mathrm{RH}=95 \%$, the relative mass gain reached 19 $\%$. This value was correlated with our gravimetric measurements and previous works realized on similar materials (Gouanvé et al., 2007; Stamboulis, Baillie \& Peijs, 2001). The desorption kinetic present a sigmoidal shape that could be interpreted as being the consequence of a delay time in the establishment of a water concentration equilibrium at the surface of the studied fibre (similar behaviour was monitored for the others studied fibres (data not shown). At the end of the experiment the initial weight of the fibre was not recovered. A relative mass loss of about $8 \%$, compared to the initial weight, was observed. Such mass loss could be attributed to the existence of water content in fibres at ambient relative humidity (Baley, Morvan, \& Grohens, 2005).

Our experimental data could be well fitted by the Carter and Kibler model as described in Célino and colleagues previous works (Célino et al., 2013). In this model, the moisture absorption is described quantitatively by assuming that the absorbed moisture consisted of both a mobile (i.e. free water) and a bound phase (i.e. bound water). Molecules of the mobile phase should diffuse spontaneously with a concentration and stress independent diffusion coefficient $\mathrm{D}_{\gamma}$, and should be absorbed with a probability per unit time $\gamma$ at certain molecular sites of the polymer fibre. On the other hand, molecules of the bound phase should be released, becoming thereby mobile, with a probability per unit time $\beta$. This assumption was supported by the qualitative FTIR spectral data recorded during the diffusion kinetic (figure $5 b)$. Indeed, during the monitoring of the water desorption phenomenon, the main impacted regions (diminishing absorbance) corresponded to the vibration regions of both the free and bound water (3600-3200 $\left.\mathrm{cm}^{-1}\right)$ and the free water uniquely $\left(1635 \mathrm{~cm}^{-1}\right)$. Those experimental observations strengthen the assumption of the Carter and Kibler model, suggesting that two kind of water population coexisted in the fibres during absorption or desorption. Water should bind to specific sites as hydroxyl or carboxyl functions and free mobile water could diffuse inside the fibre porous structure.

The curve obtained by gravimetric measurement or determined by the PLS-R model based on ATR-FTIR measurement, are in a good agreement. A slight slope difference could be however observed during the transient step. This could be attributed to the fact that the two kinetics were not strictly monitored in identical conditions (section 2.3.2). In gravimetric 
475

476

477

478

479

measurement, bundle of fibres were free of physical constraints whereas in ATR-FTIR measurements, fibres were pressed above the measurement crystal. The pressure applied by the ATR hammer could accelerate the diffusion of the water from the fibre sample, explaining therefore the observed deviation.
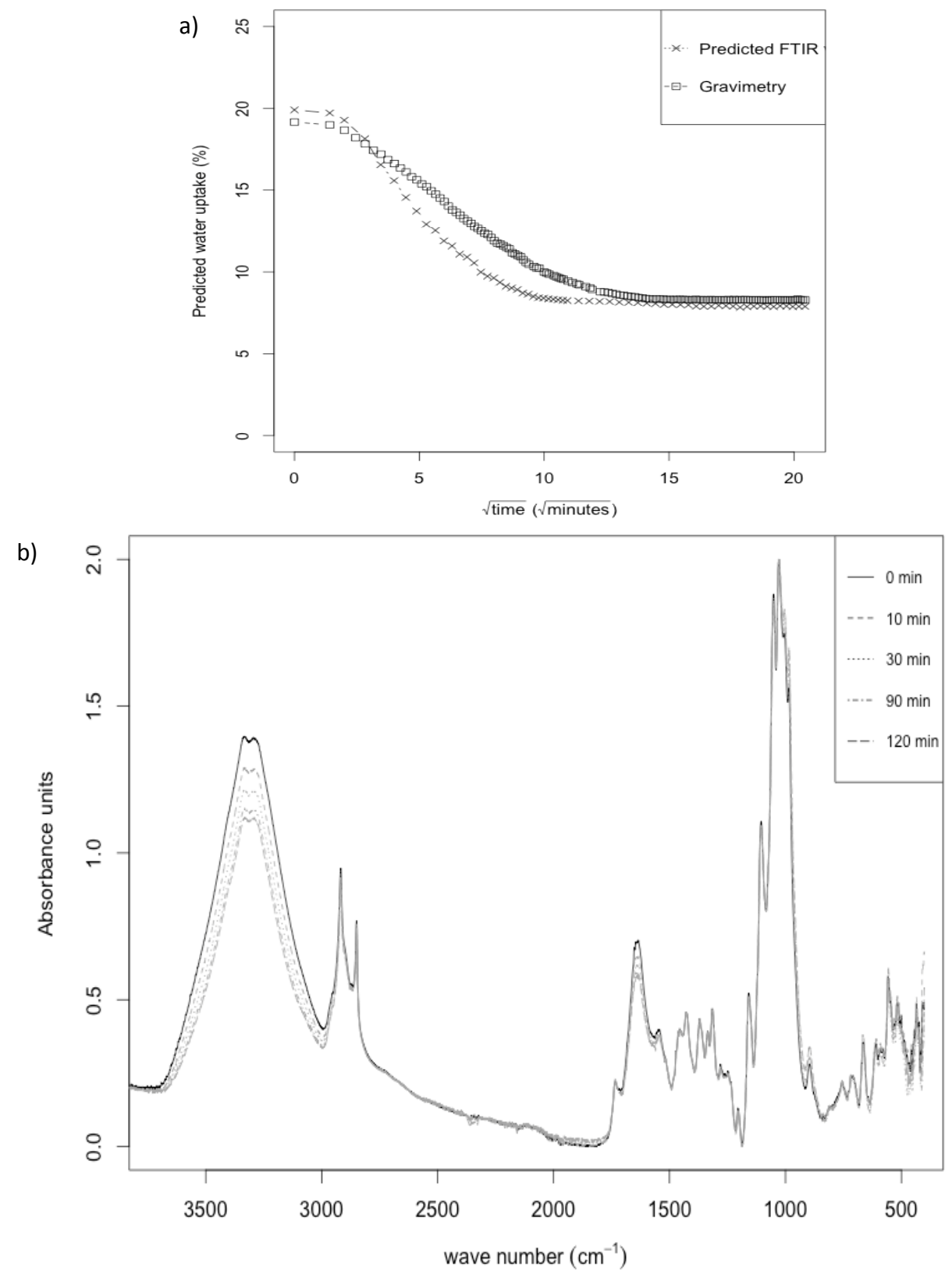

Figure 5: Quantitative and qualitative monitoring of the water diffusion kinetic for the sisal fibre. (a) Desorption kinetics monitored by FTIR PLS-R prediction and gravimetric approaches. (b) FTIR spectra recorded during kinetic monitoring for time =0, 10, 30, 90 and $120 \mathrm{~min}$.

\section{Conclusion}

In the emerging field of composite biomaterials, natural fibres promise an immense potential of application. However, their strong hygroscopic behavior requires the understanding of the moisture sorption mechanisms. Their water content is also of great importance since it could 
drive the final properties of the composites where they are fitted in. The aim of this work was to develop a quick and easy method based on ATR-FTIR spectroscopy to characterize qualitatively and quantitatively the water sorption phenomenon directly on raw fibres.

First, our results indicated that it was necessary to develop an approach per fibre since the three tested biomaterials presented their own FTIR signature and therefore their own chemical fingerprint. Hydroxyl and carboxyl molecular sites of the fiber polymer were found to be directly impacted by the water uptake, and confirmed the results observed with less structured cellulosic material (Berthold et al., 1998, Olsson \& Salmén, 2004). Second, the FTIR PLS-R models developed for each fibre, in order to quantify their water content, showed very good predictive abilities when compared to the reference method (gravimetric measurements). They were used to monitor accurately sorption isotherms whereas univariate FTIR models could not fit it correctly as depicted by Olsson and colleagues (Olsson \& Salmén, 2004). The proposed approach could also supply readily interpretable kinetic data by monitoring continuously the water desorption. The main advantage is the interpretation of kinetics at the molecular level by studying the impacted spectral bands. More detailed analysis (deconvolution of the $\mathrm{OH}$ valency band) could lead to the discrimination of the free and the bound water as described in Cotugno and colleagues previous work on an epoxy system (Cotugno et al., 2005).

Moreover, our results indicated that the information collected at the surface of the fibre could be efficiently correlated to a global method based on volumetric measurements (i.e. gravimetry). The multivariate approach used to characterize the water sorption dynamically or statically could explain the quality of the results and should encourage the use of such a direct and rapid approach for water sorption mechanism study on other cellulosic biomaterials.

\section{References:}

ASTM Committee E13 on Molecular Spectroscopy and Chromatography. (2005). Standard practices for infrared multivariate quantitative analysis. E 1655-05, 1-29.

Adibi, N., Cabrales, L., \& Haigler, C.H. (2013). Changes in the cell wall and cellulose content of developing cotton fibers investigated by FTIR spectroscopy. Carbohydrate Polymers. In press.

Alix, S., Philippe, E., Bessadok, A., Lebrun, L., Morvan, C., \& Marais, S. (2009). Effect of chemical treatments on water sorption and mechanical properties of flax fibres. Bioresource and Technology, 100, 4742-4749.

Baley, C., Morvan, C., \& Grohens, Y. (2005). Influence of absorbed water on the tensile strength of flax fibers. Macromolecules Symposium, 222, 195-202.

Berthold, J., Olsson, R.J.O., \& Salmén, L. (1998). Water sorption to hydroxyl and carboxylic acid groups in Carboxymethylcellulose (CMC) studied with NIR-spectroscopy. Cellulose, 5, 280-298. 
534 Bessadok, A., Langevin, D., Gouanvé, F., Chappey, C., Roudesli, S., \& Marais, S. (2009).

535 Study of water sorption on modified Agave fibres. Carbohydrate Polymers, 76, 74-85.

536

537

538

539

540

541

542

543

544

545

546

547

548

549

550

551

552

553

554

555

556

557

558

559

560

561

562

563

564

565

566

567

568

569

570

571

572

573

574

575

576

577

578

579

580

581

582

583

Bledzki, A.K., \& Gassan, J. (1999). Composites reinforced with cellulose based fibres, Progress in Polymer Science, 24, 221-274.

Célino, A., Fréour, S., Jacquemin, F., \& Casari, P. (2013). Characterization and modeling of the moisture diffusion behaviour of natural fibres. Journal of Applied Polymer Science, In press. DOI: 10.1002/app.39148.

Chalmers, J.M., \& Dent, G. (1997). Industrial Analysis with Vibrational Spectroscopy, RCS.

Cotugno, S., Larobina, D., Mensitieri, G., Musto, P., \& Ragosta, G. (2001). A novel spectroscopic approach to investigate transport processes in polymers: the case of water epoxy system. Polymer, 42, 6431-6438.

Cotugno, S., Mensitieri, G., Musto, P., \& Sanguigno, L. (2005). Molecular interactions in and transport properties of densely cross linked networks: a time resolved FTIR spectroscopy investigation of the epoxy/H2O systems. Macromolecules, 38, 801-811.

De Rosa, I.M., Kenny, J.M., Puglia, D., Santulli, C., \& Sarasini, F. (2010). Morphological, thermal and mechanical characterization of okra (Abelmoschus esculentus) fibres as potential reinforcement in polymer composites. Composite Science and Technology, 70, 116-122.

Di Giambattista, L., Pozzi, D., Grimaldi, P., Gaudenzi, S., Morrone, S., \& Castellano, A. C. (2011). New marker of tumor cell death revealed by ATR-FTIR spectroscopy. Analytical and Bioanalytical Chemistry, 399, 2771-2778.

Dhakal, H.N., Zhang, Z.Y., \& Richardson, M.O.W. (2007). Effect of water absorption on the mechanical properties of hemp fibre reinforced unsaturated polyester composites, Composite Science and Technology, 67, 1674-1683.

Feng, J., Berger, K.R., \& Douglas, E.P. (2004). Water vapor transport in liquid crystalline and non-liquid crystalline epoxies. Journal of Materials Science, 39, 3413-3423.

Fengel, D. (1992). Characterization of Cellulose by Deconvoluting the OH Valency Range in FTIR Spectra. Holsforshung, 46, 283-288.

Fieldson, G.T., \& Barbari, T.A. (1993). The use of FTIR-ATR spectroscopy to characterize penetrant diffusion in polymers. Polymer, 34, 1146-1153.

FitzPatrick, M., Champagne, P., \& Cunningham, M. (2012). Quantitative determination of cellulose dissolved in 1-ethyl-3-methylimidazolium acetate using partial least squares regression on FTIR spectra. Carbohydrate Polymers, 87, 1124-1130.

Gouanvé, F., Marais, S., Bessadok, A., Langevin, D. \&, Métayer, M. (2007). Kinetics of water sorption in flax and PET fibers. European Polymer Journal, 43, 586-598.

Griffiths, P.R., \& Haseth, J.A. (2007). Fourier Transformed Infrared Spectrometry (pp.321329), Second edition. John Wiley \& Sons, Inc. 
584

585

586

587

588

589

590

591

592

593

594

595

596

597

598

599

600

601

602

603

604

605

606

607

608

609

610

611

612

613

614

615

616

617

618

619

620

621

622

623

624

625

626

627

628

629

630

631

632

Hinterstoisser, B., \& Salmén, L. (1999). Two-dimensional step-scan FTIR: a tool to unravel the OH-valency-range of the spectrum of Cellulose I. Cellulose 6, 251-263.

Kondo, T. (1997). The assignment of IR absorption bands due to free hydroxyl groups in cellulose. Cellulose, 4, 281-292.

Laity, P.R., \& Hay, J.N. (2000). Measurement of water diffusion through cellophane using attenuated total reflectance-fourier transform infrared spectroscopy. Cellulose, 7, 387-397.

Liang, C.Y., \& Marchessault, R.H. (1959). Infrared spectra of crystalline polysaccharide. I. Hydrogen bonds in native cellulose. Journal of Applied Polymer Science, 39, 269-278.

Lê, S., Josse J., \& Husson, F. (2008). FactoMineR: A R Package for Multivariate Analysis. Journal of Statistical Software, 25, 1-18.

Marchessault, R.H. (1962). Application of infra-red spectroscopy to cellulose and wood polysaccharides. Pure Applied Chemistery, 5, 107-130.

Mecozzi, M., Pietroletti, M., \& Tornambe, A. (2011). Molecular and structural characteristics in toxic algae cultures of Ostreopsis ovata and Ostreopsis spp. evidenced by FTIR and FTNIR spectroscopy. Spectrochimica Acta Part A: Molecular and Biomolecular Spectroscopy, 78, 1572-1580.

Mijovic, J., \& Zhang, H. (2003). Local dynamics and molecular origin of polymer networkwater interactions as studied by broadband dielectric relaxation spectroscopy, FTIR and molecular simulations. Macromolecules, 36, 1279-1288.

Musto, P., Ragosta, G., \& Mascia, L. (2000). Vibrational spectroscopy evidence for the dual nature of water sorbed into epoxy resins. Chemistery of Material, 12, 1331-1341.

Murphy, D., \& Pinho, M.N. (1995). An ATR-FTIR study of water in cellulose acetate membranes prepared by phase inversion. Journal of Membrane Science, 106, 245-257.

Nelsson, M.L., \& O’Connor, R.T. (1964). Relation of Certain Infrared Bands to Cellulose Crystallinity and Crystal Lattice Type. Part I. Spectra of Lattice Types I, 11, I11 and of Amorphous Cellulose. Journal of Applied Polymer Science, 8, 1311-1324.

Olsson, A.M., \& Salmén, L. (2004). The association of water to cellulose and hemicelluloses in paper examined by FTIR spectroscopy. Carbohydrate Research, 339, 813-818.

Park, G. S. (1986). Transport principles: Solution, diffusion and permeation in polymer membranes. In P. M. Bungay et al. (Eds.). Synthetic membranes: Science, engineering and applications (57). Holland: Reidel Pub.

Parker, F.S. \& Roy, A. (1966). Infrared spectra of carbohydrates (700 - $\left.250 \mathrm{~cm}^{-1}\right)$ determined by both Attenuated Total Reflectance and Transmission techniques. Applied Spectroscopy, 20, 384-388. 
633

634

635

636

637

638

639

640

641

642

643

644

645

646

647

648

649

650

651

652

653

654

655

656

657

658

659

660

661

662

663

664

665

666

667

668

669

670

671

672

673

674

675

676

677

678

679

680

Placet, V., Cisse, O., \& Boubakar, L. (2012). Influence of environmental relative humidity on the tensile and rotational behaviour of hemp fibres. Journal of Materials Science, 47, 34353446

Popineau, S., Rondeau-Mouro, C., Sulpice-Gaillet, C., \& Martin E.R.S. (2005). Free/bound water absorption in an epoxy adhesive. Polymer, 46, 10733-10740.

Satyanarayana, K.G., Arizaga, G.G.C., \& Wypych, F. (2009). Biodegradable composites based on lignocellulosic fibers - An overview. Progress in Polymer Science, 34, 982-1021.

Stamboulis, A., Baillie, C.A., \& Peijs, T. (2001). Effect of environmental conditions on mechanical and physical properties of flax fibres. Composites Part A, 32, 1105-1115.

Suddell, B.C., \& Evans, W.J. (2005). Natural Fiber Composites in Automotive Applications. In Mohanty, A.K., Misra, M. \& Drzal, L.T. (Eds.), Natural Fibers in Biopolymers \& Their BioComposites (pp. 231-259). CRC Press.

Summerscales, J., Dissanayake, N.P.J., Virk, A.S., \& Hall, W. (2010). A review of bast fibres and their composites. Part I-Fibres as reinforcements. Composites : Part A, 41, 1329-1335.

Symington, M.C., Banks, W.M., David, W.O, \& Pethrick, R.A. (2009). Tensile Testing of Cellulose Based Natural Fibers for Structural Composite Applications. Journal of Composite Materials, 43, 1083-1108.

Van den Berg, R. A., Hoefsloot, H. C., Westerhuis, J. A., Smilde, A. K., \& Van Der Werf, M. J. (2006). Centering, scaling, and transformations: improving the biological information content of metabolomics data. BMC Genomics, 7, 142-153.

Wambua, P., Ivens, J., \& Verpoest, I. (2003). Natural fibres: can they replace glass in fibre reinforced plastics? Composite Science Technology, 63, 1259-1264.

Westerhuis, J. A., Hoefsloot, H. C. J., Smit, S., Vis, D. J., Smilde, A. K., Velzen, E. J. J., John P. M., van Duijnhoven, Ferdi A. van Dorsten. (2008). Assessment of PLSDA cross validation. Metabolomics, 4, 81-89.

Wold, S., Sjöström, M., \& Eriksson, L. (2001). PLS-regression: a basic tool of chemometrics. Chemometrics and Intelligent Laboratory Systems, 58, 109-130.

Xia, J., Mandal, R., Sinelnikov, I.V., Broadhurst, D., \& Wishart, D.S. (2012). MetaboAnalyst 2.0-a comprehensive server for metabolomic data analysis. Nucleic Acids Research, 40, 127133.

Xie, Y., Hill, C.A.S., Jalaludin, Z., Curling, S.F., Anandjiwala, R.D., Norton , A.J. \& Newman, G. (2011). The dynamic water vapour sorption behaviour of natural fibres and kinetic analysis using the parallel exponential kinetics model. Journal of Material Science, 46, 479-489.

\section{List of figures}

Figure 1: PLS-DA score plot of second derivative FTIR signatures of the three model fibres for three RH conditions. 
The legend in the upright corner details the nature of the model fibre and the values of the tested RH \% (as suffixes). The classification model used here presented the following characteristics i.e. $\mathrm{R}^{2}(\mathrm{Y})=0,989$ and $\mathrm{Q}^{2}=0,869$ and was validated using 20 permutations $\left(\mathrm{R}^{2}(\mathrm{Y})\right.$ int $=0,555$ and $\mathrm{Q}^{2}$ int $\left.=-0,343\right)$.

Figure 2: Infrared spectra bands impacted by increasing relative humidity for sisal fibre. For clarity, only sisal fibre has been presented and only three relative humidities are shown for three replicates (10\%; $50 \%$ and $85 \%)$. p-values scores, indicating significant impact of the water uptake on the FTIR bands, were marked using red dots.

Figure 3: Cross-validation (a) and test set validation (b) results of water uptake prediction for sisal fibre.

The PLS-R predictions of the water content obtained with the FTIR approach were compared here to the gravimetric measurements. The water uptake is expressed in \% of the dry weight of the sisal fibre. The coefficients of determination (\%) of the regression lines (indicated in dotted lines) are indicated in the upper left corner.

Figure 4: Comparison of sorption isotherm for sisal fibre using FTIR and gravimetric approaches. (a) Sorption isotherm plotted for the sisal fibre according to the measurement method (FTIR univariate, FTIR multivariate, gravimetry). (b) Comparison performed between normalized FTIR measurements and normalized gravimetric reference method measurements (FTIR univariate-gravimetry: $\mathrm{O}$, FTIR multivariate-gravimetry $: \triangle$ ). $\mathrm{R}^{2}$ stands for coefficient of determination (\%).

Figure 5: Quantitative and qualitative monitoring of the water diffusion kinetic for the sisal fibre. (a) Desorption kinetics monitored by FTIR PLS-R prediction and gravimetric approaches. (b) FTIR spectra recorded during kinetic monitoring for time $=0$, 10, 30, 90 and 120 min.

\section{List of tables}

Table 1: Assignment of the main absorption bands in FTIR spectra of sisal, flax and hemp fibres. Sisal FTIR data were interpreted according to investigations issued from (Adibi, Cabrales, \& Haigler, 2013; De Rosa, Kenny, Puglia, Santulli, \& Sarasini, 2010; Liang \& Marchessault, 1959; Nelsson \& O’Connor, 1964).

Table 2: PLS-R quality parameters for cross and test set validation for the models selected for the three studied fibres.

RMSECV stands for Root Mean Square Error of Cross Validation. RMSEP stands for Root Mean Square Error of Prediction. $\mathrm{R}^{2}$ is the coefficient of determination. 
- Effect of moisture sorption on plant fibres was investigated by FTIR spectroscopy.

723 - Hydroxyl and carboxyl moieties are specific sites for water sorption.

724 - Partial least squares regression quantified the water content of natural fibres.

725 - Models fitted the sorption isotherms and diffusion kinetics.

726

727

728 